\section{Contrast Media Volume is Significantly Related to Patient Lung Volume during Computed Tomography Pulmonary Angiography When Employing a Patient- specific Contrast Protocol}

\author{
Charbel Saade ${ }^{1}$, Youssef Ghosn², Ghina Alfout ${ }^{2}$, Mustafa Zuhair Mahmoud ${ }^{3}$, \\ Mohammad Ahmmad Rawashdeh ${ }^{4}$, Lina Karout ${ }^{1}$, \\ Diamond Ghieh², Fadi El-Merhi ${ }^{2}$
}

\begin{abstract}
${ }^{1}$ Faculty of Health Sciences, American University of Beirut Medical Center, Beirut 1107 2020, Lebanon, 2Department of Diagnostic Radiology, American University of Beirut Medical Center, Beirut 1107 2020, Lebanon, ${ }^{3}$ Department Radiology and Medical Imaging, College of Applied Medical Sciences, Salman bin Abdulaziz University, Al-Kharj 11942, Saudi Arabia, ${ }^{4}$ Department of Allied Medical Sciences, Jordan University of Science and Technology, Irbid 22110, Jordan
\end{abstract}

Address for correspondence: Fadi El-Merhi, Department of Imaging, Dana-Farber Cancer Institute and Harvard Medical School, Boston, MA, USA; Department of Radiology, Brigham and Women's Hospital and Harvard Medical School, Boston, MA, US. E-mail: fel-merhi@bwh.harvard.edu

\section{Abstract}

Purpose: The purpose of this study is to investigate the relationship between contrast media volume (CMV) and patient lung volume when employing a patient-specific contrast media formula during pulmonary computed tomography angiography (CTA). Materials and Methods: Institutional review board approved this retrospective study. CTA of the pulmonary arteries was performed on 200 patients with suspected pulmonary embolism. The CMV was calculated by employing a patient-specific contrast formula. Lung volume was quantified employing semi-automated lung software that calculated lung volumes (IntellispacePhilips). The mean cross-sectional opacification profile of central and peripheral pulmonary arteries and veins was measured for each patient and arteriovenous contrast ratio (AVCR) calculated for each lung segment. Mean body mass index and lung volume were quantified. Receiver operating characteristic (ROC) and visual grading characteristics (VGC) measured reader confidence in emboli detection and image quality, respectively. Inter- and intra-observer variations were investigated, employing Cohen's kappa methodology. Results: Results showed that the mean pulmonary arterial opacification of the main pulmonary circulation (343.88 \pm 73 Hounsfield units [HU]), right lung; upper ( $316.51 \pm 23 \mathrm{HU})$, middle $(312.5 \pm 39 \mathrm{HU})$, and lower $(315.23 \pm 65 \mathrm{HU})$ lobes and left; upper $(318.76 \pm 83 \mathrm{HU})$ and lower $(321.91 \pm 12 \mathrm{HU})$ lobes. The mean venous opacification of all pulmonary veins was below $182 \pm 72 \mathrm{HU}$. AVCR was observed at all anatomic locations $(P<0.0002)$, where this ratio was calculated. Moreover, larger volumes of contrast significantly correlated with larger lung volumes $(r=0.89, P<0.03)$ and radiation dose $(P<0.03)$. VGC and ROC analysis demonstrated an increased area under the curve: 0.831 and 0.99 , respectively $(P<0.02)$. Inter-observer variation was observed as excellent $(\kappa=0.71)$. Conclusion: We conclude that increased $C M V$ is significantly correlated to increased patient lung volume and radiation dose when employing a patient-specific contrast formula. The effects patient habitus is highlighted.

Keywords: Computed tomography, Contrast media, Contrast protocol, Lung volume,

Pulmonary angiography

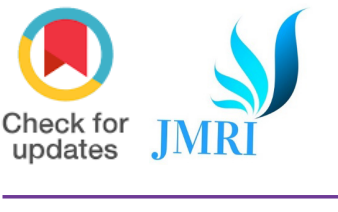

How to cite this article: Saade C, Ghosn Y, Alfout G, Mahmoud MZ, Rawashdeh MA, Karout L, Ghieh D, El-Merhi F. Contrast Media Volume is Significantly Related to Patient Lung Volume during Computed Tomography Pulmonary Angiography When Employing a Patient-specific Contrast Protocol. J Med Res Innov. 2020;4(2):e000207.

DOI: $10.32892 /$ jmri.207

Publication history: Received: 13-02-2020 Accepted: 23-04-2020

Published: 08-05-2020

Editor: Dr. Varshil Mehta

Copyright: Saade $C$, Ghosn Y, Alfout G, Mahmoud MZ, Rawashdeh MA, Karout L, Ghieh D, El-Merhi F. This is an open-access article distributed under the terms of the Creative Commons Attribution License CC-BY 4.0., which permits unrestricted use, distribution, and reproduction in any medium, provided the original author and sources are credited.

Funding: NIL

Conflict of Interest: Charbel Saade declares that he has no conflict of interest. Youssef Ghosn declares that he has no conflict of interest. Ghina Alfout declares that she has no conflict of interest. Mustafa Zuhair Mahmoud declares that he has no conflict of interest. Mohammad Ahmmad Rawashdeh declares that he has no conflict of interest. Lina Karout declares that she has no conflict of interest. Diamond Ghieh declares that she has no conflict of interest. Fadi ElMerhi declares that he has no conflict of interest. 


\section{Introduction}

Although the gold standard in pulmonary embolism $(\mathrm{PE})$ diagnosis is still angiopulmonography, computed tomography pulmonary angiography (CTPA) protocols have witnessed a surge in thrombus detection at specificity and sensitivity approaching $100 \%$ with additional benefits of noninvasiveness and accessibility. ${ }^{[1]}$ Since this efficacy is largely dependent on effective contrast media volume (CMV) administration, studies have been extensively conducted on contrast administration protocols. ${ }^{[2-8]}$ These studies have found that visualization of the pulmonary vasculature is significantly improved when using a simple patientspecific formula, ${ }^{[1,7,9]}$ which also allows CMV to be reduced along with the potential risk of contrastinduced nephropathy. ${ }^{[1]}$

Studies performed on adult patients with normal pulmonary functioning demonstrated an air/tissue relationship approaching a 7:3 ratio of air to lung parenchyma; ${ }^{[10]}$ other studies noticed that an increase in total lung volume is concomitant with an increase in the volume of pulmonary blood and pulmonary extravascular water. ${ }^{[11]}$ To the best of our knowledge, there have been no studies to date that compares $\mathrm{CMV}$ with patient lung volume employing a reduced patient-specific formula. The aim of the study is to investigate the relationship between $\mathrm{CMV}$ and patient lung volume when employing a patient-specific contrast media formula during CTPA. Our results could be used as a foundation for further research on lung volume-specific CTPA protocols.

\section{Materials and Methods Study population}

The institutional review board (IRB) approved this study and written informed consent was waived since all studies were clinically indicated and patient data were evaluated retrospectively. Two hundred consecutive patients with high clinical suspicion of acute PE were examined over a 6-month period between August 2018 and January 2019. The indication for CTPA was suspected PE based on clinical information (chest pain, dyspnea, hypoxia, calf pain or known deep vein thrombosis, and risk factors for $\mathrm{PE}$ ) and/or laboratory information (positive d-dimer $>0.8 \mathrm{mg} / \mathrm{L}$ ). All patients with a positive $\mathrm{PE}$ in this study received anticoagulation therapy and due to the nature of the suspected condition, all patients were scanned. Inclusion criteria were patients with suspected PE as per the criteria mentioned above, who underwent CTPA. Patients with no suspected PE or patient with PE who did not undergo CTPA were excluded from the study. There were no patients in the study that had renal insufficiency and/or contraindications to iodinated contrast media. The diagnosis and treatment were in adherence to the American College of Cardiology (ACC) guidelines for the diagnosis and management of PE.

\section{Ethical approval}

All procedures performed in studies involving human participants were in accordance with the ethical standards of the American University of Beirut's IRB and with the 1964 Helsinki declaration and its later amendments or comparable ethical standards. Informed consent was waived since all studies were clinically indicated; patient data contained no identifiable information and was evaluated retrospectively.

\section{Image acquisition}

CTPA was performed using a 256-channel computed tomography scanner (Philips Brilliance iCT, Philips Healthcare, The Netherlands). Patients were positioned supine with arms resting on the gantry above the head. Anterior-posterior scout was performed, with a scan range from the apices $(2 \mathrm{~cm}$ above the $\left.1^{\text {st }} \mathrm{rib}\right)$ to the diaphragm $(2 \mathrm{~cm}$ below the lowest costophrenic recess). Breath-hold, with a mouth open breathing technique, was employed to reduce hyperventilation and Valsalva. Scan range was from the lung apices to the costophrenic angles. CT scan parameters employed in each protocol were: Detector width of $256 \times 0.625 \mathrm{~mm}$, pitch of 0.981:1 ratio, rotation time of $0.27 \mathrm{~s}$, $100 \mathrm{kVp}, 140 \mathrm{~mA}$, with $\mathrm{x}, \mathrm{y}$ and $\mathrm{z}$-axis modulation (DoseRight), craniocaudal scan direction, and model-based iterative reconstruction (IMR2).

\section{Contrast media administration}

\section{Contrast bolus geometry}

Vessel opacification for all cases was measured by placing a region of interest (ROI) over the main pulmonary trunk. A time attenuation curve in Hounsfield units (HU) was calculated and the desired peak opacification was recorded. The patient-specific protocol employed the test bolus technique ${ }^{[1]}$ where the ROI was plotted inside the 
main pulmonary trunk with a small amount of contrast material $(5 \mathrm{~mL})$ injected at the same rate as the main bolus. This ROI assessed the time to peak (TTP) and determined the arteriovenous circulation time for pulmonary vasculature. ${ }^{[1,7,12]}$

\section{Contrast media acquisition}

Contrast and saline chaser were injected with an automated dual-barrel power injector (CT Emotion, Ullrich, Germany) through a $20 \mathrm{G}$ venous catheter in the right arm. ${ }^{[13,14]}$ The patient-specific contrast formula employed iodinated contrast (Omnipaque $350 \mathrm{mgl} / \mathrm{mL}$; General Electric, USA) intravenously injected at a flow rate of $4.5 \mathrm{~mL} / \mathrm{s}$. Contrast media volume was calculated according to an empirically derived formula: ${ }^{[15]} \mathrm{CV}=(\mathrm{ST}+\mathrm{TTP}-\mathrm{OVWP}) \times \mathrm{FR}$. Where ST is the scan time (s), TTP (s) is as described above, OVWP is the optimal venous washout phase (6 s), and FR is the flow rate $(\mathrm{mL} / \mathrm{s})$. ST differs for each patient based on the distance between the apices and diaphragm of the thorax. Patients were excluded if they were unable to have a right-sided injection site in the cubital fossa with a flow rate of $4.5 \mathrm{~mL} / \mathrm{s}$.

\section{Radiation dose measurement}

For each of the CT scans, an individual effective dose $\left(\mathrm{E}_{\mathrm{ff}}[\mathrm{mSv}]\right)$ was calculated from the doselength products (DLP [mGy $\times \mathrm{cm}]$ ), which were recorded from the patient protocol. A normalized conversion factor $(\mathrm{k}[\mathrm{mSv} / \mathrm{mGy} \times \mathrm{cm}])$ for the chest $-0.014 \mathrm{mSv} / \mathrm{mGy} \times \mathrm{cm}-$ was used to calculate the $\mathrm{E}_{\mathrm{ff}} \cdot{ }^{[16]} \mathrm{E}_{\mathrm{ff}}=\mathrm{DLP} \times \mathrm{k}^{\left[{ }^{[4]}\right.}$

\section{Image assessment}

Technical inclusion criteria ensuring correct scan range and anatomical inclusion of the origin, pathway, and termination of the pulmonary vasculature were applied to all cases by two expert radiologists (not included in the study proper). Quantitative measurements of all images were performed using a primary reporting workstation (IntelliSpace, Philips Healthcare, Netherlands) with a GSDF-calibrated 3 megapixel monitor. Illumination was adjusted at 25-32 lux, ${ }^{[17]}$ with a calibrated photometer (Chroma meter CL-200).

\section{Quantitative analysis}

Opacification in HU was measured for all cases in the trans-axial images within the largest circular
ROI that would fit within the lumen and exclude the vessel wall. The mean cross-sectional opacification profile of 8 central segments and 11 peripheral pulmonary arteries were measured. In cases where PE was identified, care was taken not to include the emboli within the measurements. Arterial and venous measurements took place at the heart, pulmonary trunk, segmental, and subsegmental pulmonary vasculature and each measurement were no $<2 \mathrm{~mm}$ in diameter [Figure 1]. The location of the arteries and veins was as follows: Central pulmonary vasculature (trunk, right and left pulmonary arteries, and left superior and inferior pulmonary veins); pulmonary segments; right upper lobe (anterior and posterior), right middle lobe (lateral and medial), right lower lobe (anterior and posterior basal), left upper lobe (apicoposterior and inferior lingular), and left lower lobe (anteromedial basal and posterior basal); and superior vena cava (SVC).

Adjacent to the arterial pathways, the venous measurements were performed in the same axial plane. Image contrast between arteries and veins was expressed as a ratio of HU values (artery/ vein) at each anatomical level and denoted as the arteriovenous contrast ratio (AVCR). ${ }^{[18]}$

Lung volume parenchyma and airway volumes were measured by quantitative volumetric analysis [Figure 2] on the CTPA imaging sequence using the Philips IntelliSpace lung segmentation software (v6.0.3.12200, Best, The Netherlands).

\section{Diagnostic efficacy}

The multi-reader analysis consisted of two cardiothoracic radiologists who had been certified

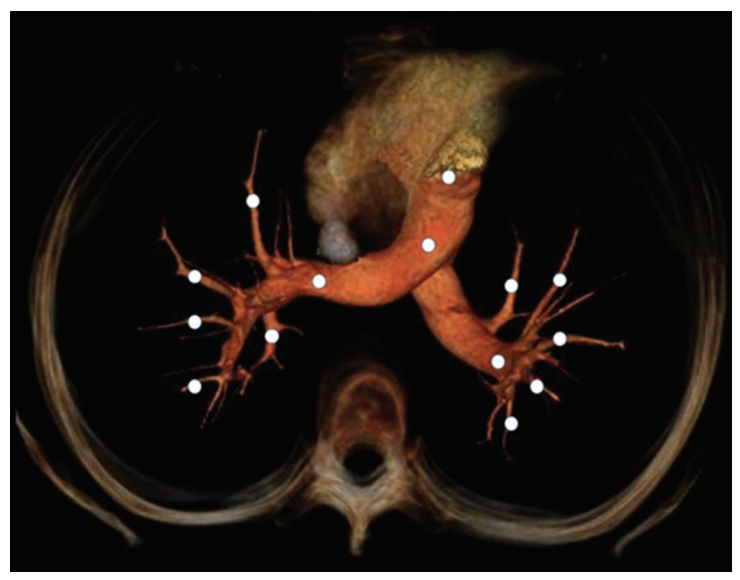

Figure 1: Anatomical location of measurements of the pulmonary vasculature 


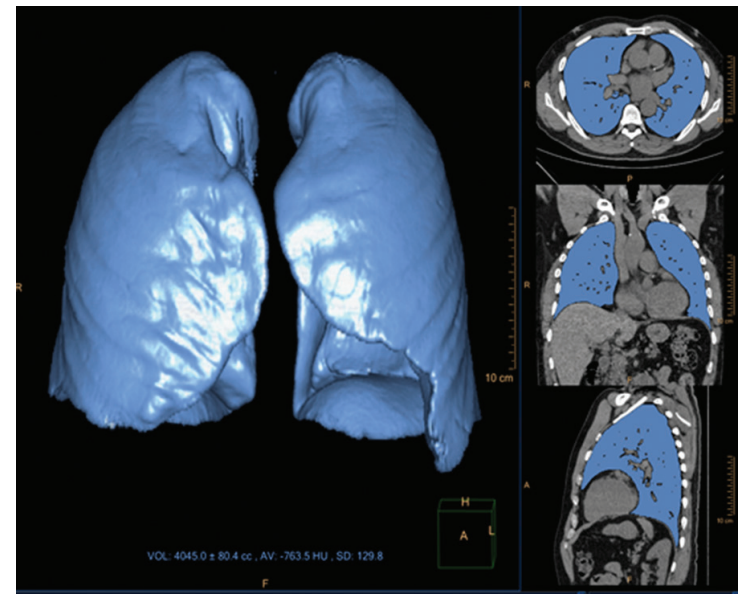

Figure 2: Lung volume analysis

by the American Board of Radiology and The Royal College of Radiologists for a mean number of 8.9 years (minimum, 3 years; maximum, 18 years). All reviewers were specialists in cardiothoracic imaging and each observer was allowed to manipulate the window level of the images.

\section{Receiver operating characteristic (ROC) analysis}

ROC methodology was employed to illustrate radiologist confidence intervals to detect pathology. A score of 1-2 was assigned to each image, where 1 indicates positive for pathology detection and 2 indicates negative for pathology detection of PE. All cases were randomly allotted with the number of normal $(n=87)$ and abnormal $(n=113)$ cases. The abnormal cases demonstrated an array of vascular disease that was defined by two radiologists' reports (based on complete patient series, previous and subsequent examinations, and clinical indications). All pathology was visible on the transaxial images and the prevalence of pathology was not revealed to the observers. Technical criteria ensuring correct scan range and anatomical inclusion were considered (not included in the study proper) to ensure that all images displayed an acceptable level of quality before they were included in the analysis.

\section{Visual grading characteristics (VGC) analysis}

The VGC method of Bath and Mansson ${ }^{[19]}$ was used to illustrate viewer assessment of image quality based on the visibility of normal anatomy. Specifically, for this work, the presence of contrast media filling was recorded for a pulmonary arterial system using a five-point classification scale where score 1 indicated no contrast media filling within the pulmonary vasculature and 5 represented complete filling.

\section{Inter-and intra-reader variability}

The inter- and intra-observer agreements were calculated using Cohen $\kappa$ analysis. A $\mathrm{k}$ value 0.60 to $1,0.41$ to $0.60,0.21$ to 0.40 , and $<0.20$ was considered excellent, moderate, fair, and poor agreement, respectively.

\section{Statistical analysis}

Continuous variables were described with mean \pm standard deviation (SD). The analysis of variance (ANOVA) was used to compare the means across the tertiles of contrast volume. The association between contrast volume and measured variables was evaluated through simple linear regression. Analyses were conducted using SPSS 22 for Windows (SPSS Inc., Chicago, IL). Results were considered statistically significant if $P \leq 0.05$ with a $95 \%$ confidence interval and a power of 0.8 . ROC and VGC were employed to measure the confidence intervals in pathology detection and image quality, respectively. Jackknife free-response assessment of diagnostic systems continues to gain acceptance in areas related to the detection, localization, and classification of one or more "abnormalities" within a subject.

\section{Results}

\section{Patient demographics}

There was no significant change in $\mathrm{CMV}$ with gender and age, however, CMV increased with increasing body weight $(P<0.004)$ and body mass index (BMI) $(P<0.01)$ [Table 1]. In addition, the effective radiation dose also increased from low CMVs $(0-10 \mathrm{~mL})$ to higher CMVs $(60-70 \mathrm{~mL})(P<0.03)$ incrementally.

\section{Image acquisition and CMV}

There was no statistical significance in mean scan time in each contrast range; $0-10 \mathrm{~mL}:(3.22 \pm 1.2$ s) compared to $60-70 \mathrm{~mL}:(4.39 \pm 1.3 \mathrm{~s}),(P>0.05)$ [Table 1]. All patients tolerated their assigned contrast material delivery protocol without any related complications. 


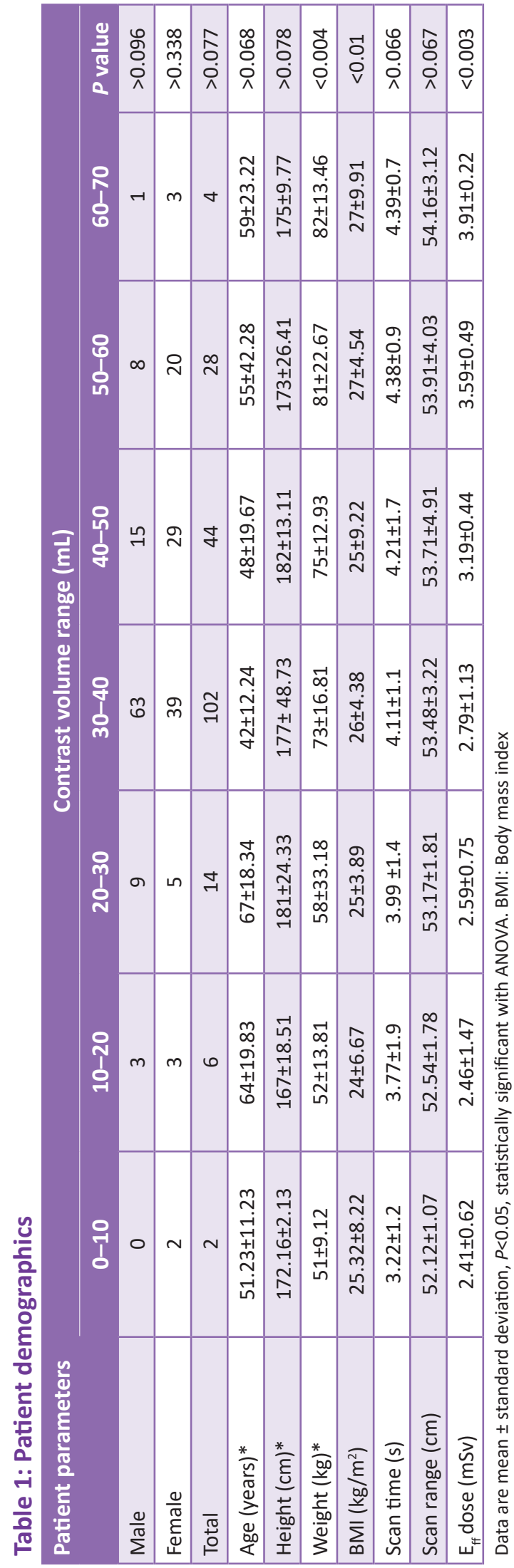

\section{Quantitative analysis}

The mean pulmonary arterial opacification of the main pulmonary circulation was (343.88 $\pm 73 \mathrm{HU})$. For the right lung, the mean arterial opacification was: Upper lobe (316.51 $\pm 23 \mathrm{HU})$, middle lobe (312.5 $\pm 39 \mathrm{HU})$, and lower lobe (315.23 $\pm 65 \mathrm{HU})$. For the left lung, the mean arterial opacification was: Upper lobe $(318.76 \pm 83 \mathrm{HU})$ and lower lobe (321.91 $\pm 12 \mathrm{HU})$ [Table 2]. The mean venous opacification of all pulmonary veins was below the threshold of $182 \pm 72 \mathrm{HU}$ [Table 2]. In addition, the opacification of the superior vena cava veins was approximately double that of the mean pulmonary artery opacification. The AVCR ranged from 2.21:1 to $3.83: 1(P<0.0010)$, which demonstrated a significant difference between arterial and venous opacification [Table 3].

\section{Lung volume versus contrast volume in patient- specific contrast formula}

When employing the patient-specific formula, an increase in CMV positively correlated with increased total lung volume $(r=0.89, P<0001)$. As expected, total lung volume increased with increasing mean anteroposterior and lateral lung diameters. Therefore, increased lung volume, anteroposterior, and lateral chest diameters are correlated with increased CMV [Table 4].

However, we further stratified CMV groups into tertiles ( 3 groups) to equally distribute the number of patients across each group, unlike in Table 4. Regression analysis was performed for each CMV group relative to lung volume. By comparing CMV $1(<32 \mathrm{~mL})$ and 2 (>33-<50 mL) demonstrated significant changes in the right and left lungs $(P<0.006)$ [Table 5]. Furthermore, when comparing Group $2(>37-<40 \mathrm{~mL})$ with Group $3(>41 \mathrm{~mL})$, statistical significance was seen with increasing lung volumes in both rights and left lung $(P<0.001)$ as well as an increase in effective radiation dose $(P<0.04)$ [Table 5]. Interestingly, after regression analysis was performed, weight and BMI had no statistically significant association with increased lung volume and CMV.

\section{Qualitative analysis}

VGC - the scores were individually graded by the two readers (R1 and R2) and were expressed as a graph [Figure 3]. The sensitivity and specificity were then compared by calculating the area under

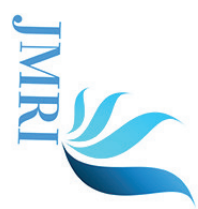


Table 2: Mean opacification (HU) of arteries and veins at each anatomical segment during CTPA

\begin{tabular}{|c|c|c|}
\hline Anatomic level & Anatomical location & Mean opacification with SD \\
\hline Pulmonary trunk & Mediastinum & $362 \pm 97$ \\
\hline Right main pulmonary artery & & $337 \pm 88$ \\
\hline Left main pulmonary artery & & $323 \pm 88$ \\
\hline Atrial vein & Mediastinum & \\
\hline Right superior & & $177 \pm 73$ \\
\hline Right inferior & & $154 \pm 67$ \\
\hline Left superior & & $182 \pm 72$ \\
\hline Left inferior & & $157 \pm 61$ \\
\hline Superior vena cava & & $606 \pm 257$ \\
\hline \multicolumn{3}{|l|}{ Pulmonary arteries } \\
\hline Right superior anterior & Right upper lobe & $320 \pm 87$ \\
\hline Right superior posterior & & $313 \pm 83$ \\
\hline Right medial & Right middle lobe & $311 \pm 93$ \\
\hline Right lateral & & $314 \pm 86$ \\
\hline Right anterior basal & Right lower lobe & $306 \pm 89$ \\
\hline Right posterior basal & & $325 \pm 95$ \\
\hline Left apicoposterior & Left upper lobe & $314 \pm 79$ \\
\hline Left inferior lingular & & $323 \pm 86$ \\
\hline Left anteromedial basal & Left lower lobe & $319 \pm 82$ \\
\hline Left posterior basal & & $323 \pm 83$ \\
\hline \multicolumn{3}{|l|}{ Pulmonary veins } \\
\hline Right superior anterior & Right upper lobe & $112 \pm 79$ \\
\hline Right superior posterior & & $122 \pm 78$ \\
\hline Right medial & Right middle lobe & $95 \pm 62$ \\
\hline Right lateral & & $101 \pm 60$ \\
\hline Right anterior basal & Right lower lobe & $92 \pm 61$ \\
\hline Right posterior basal & & $96 \pm 59$ \\
\hline Left apicoposterior & Left upper lobe & $141 \pm 80$ \\
\hline Left inferior lingular & & $146 \pm 77$ \\
\hline Left anteromedial basal & Left lower lobe & $120 \pm 61$ \\
\hline Left posterior basal & & $111 \pm 62$ \\
\hline
\end{tabular}

Data are mean \pm SD, $P<0.05$, statistically significant with ANOVA. HU: Hounsfield units, SD: Standard deviation, CTPA: Computed tomography pulmonary angiography

the curve (AUC) differences from each of the ROC curve analysis. Calculating the difference between each reader, the graphs demonstrated an AUC = 0.831 , with a $95 \%$ confidence interval of $0.71-0.89$ $(P<0.02)$.

Jackknife free-response ROC - the six-point scale demonstrated a significant difference $(P<0.001)$ between protocols with mean ROC values demonstrating strong reader confidence between each $\mathrm{CMV}$ range (95\% $\mathrm{Cl} 0.88-0.99$ ) [Figure 4]. The number of patients diagnosed with PE was 38 (19\%). Kappa analysis - CTPA yielded excellent interobserver agreement $(k=0.71)$ in all ranges. There was a strong positive relationship between mean pulmonary arterial opacification, good image quality, and reader confidence in the patient-specific protocol $(r=0.67, P<0.001)$. 
Table 3: Mean opacification (HU) profile of artery and vein at each anatomical level and the ratio of the AVCR

\begin{tabular}{|l|l|c|c|c|c|}
\hline Anatomical level & Anatomical location & Artery & Vein & Ratio & P value \\
\hline Right superior anterior & Right upper lobe & $320 \pm 87$ & $112 \pm 79$ & 2.86 & $<0.0001$ \\
\hline Right superior posterior & & $313 \pm 83$ & $122 \pm 78$ & 2.65 & $<0.0001$ \\
\hline Right medial & Right middle lobe & $311 \pm 93$ & $95 \pm 62$ & 3.27 & $<0.0001$ \\
\hline Right lateral & & $314 \pm 86$ & $101 \pm 60$ & 3.10 & $<0.0001$ \\
\hline Right anterior basal & Right lower lobe & $306 \pm 89$ & $92 \pm 61$ & 3.33 & $<0.0001$ \\
\hline Right posterior basal & & $325 \pm 95$ & $96 \pm 59$ & 3.38 & $<0.0001$ \\
\hline Left apicoposterior & Left upper lobe & $314 \pm 79$ & $141 \pm 80$ & 2.23 & 0.0002 \\
\hline Left inferior lingular & & $323 \pm 86$ & $146 \pm 77$ & 2.21 & 0.0002 \\
\hline Left anteromedial basal & Left lower lobe & $319 \pm 82$ & $120 \pm 61$ & 2.66 & $<0.0001$ \\
\hline Left posterior basal & & $323 \pm 83$ & $111 \pm 62$ & 2.91 & $<0.0001$ \\
\hline
\end{tabular}

Data are mean \pm standard deviation, $P<0.05$, statistically significant with ANOVA. HU: Hounsfield units, AVCR: Arteriovenous contrast ratio

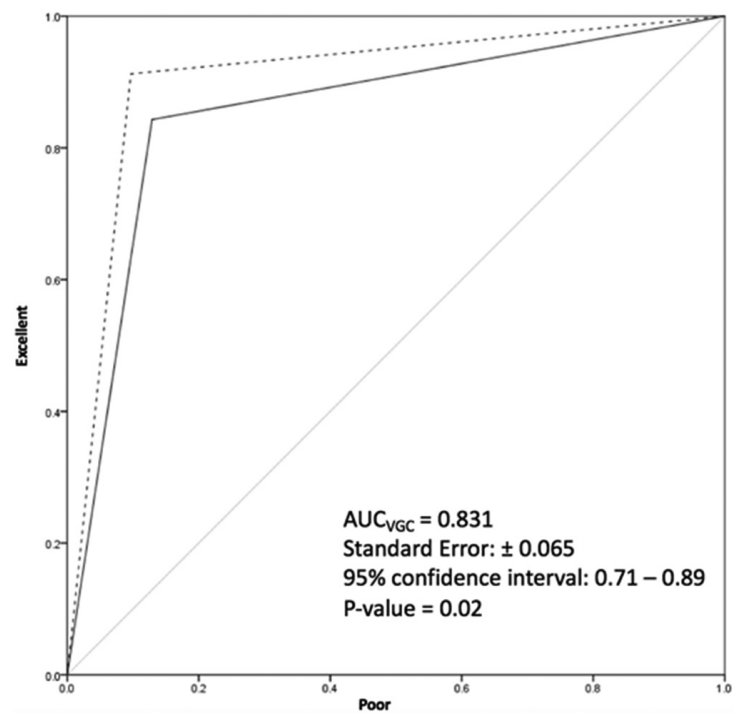

Figure 3: Visual grading characteristic curve. The graph represents positive agreement in image quality during pulmonary computed tomography angiography. Visual grading characteristics was employed to measure the confidence intervals in image quality assessment by radiologists

\section{Discussion}

There have been considerable studies carried out to reduce radiation dose through reduction of radiation output, fixed $\mathrm{CMV}$, and reduced contrast media concentrations during CTA. ${ }^{[20-22]}$ In addition, weight-based contrast media protocols have been considerably used to perform consistent optimal image quality during CTPA, but at the cost of larger CMV for considerably larger patients. ${ }^{[23]}$ However, until recently, patient-specific protocols have not been readily employed to significantly reduce contrast media dose during CTPA without compromising image quality. ${ }^{[1,7]}$ Nevertheless, to our knowledge, this is the first study to investigate a possible relationship between CMV and lung volumes, weight and $\mathrm{BMI}$, and how different CMVs could affect confidence in emboli detection and image quality when employing a patient-specific formula.

The results of this study have demonstrated a significant correlation between CMV and lung organ volumes. When this formula was applied to patients, the CMV increased with increased lung volume which might be important to maintain a good visualization of the pulmonary vasculature. Furthermore, there was no significant change in CMV with gender and age; however, CMV initially increased with increasing body weight and BMI when patients were unequally grouped into 7 protocol ranges. However, when grouped into three ranges with the same patient number, body weight and BMI did not correlate with $\mathrm{CMV}$ which is in contrast with previous findings. ${ }^{[24-28]}$ The reduced CMV did not affect pulmonary artery opacification and the sensitivity and specificity of pulmonary emboli detection as those in previously reported papers. ${ }^{[29-31]}$

There still remain controversies surrounding the effect of CMV and increased radiation dose due to the photoelectric effect. Recent studies demonstrated that the addition of contrast media 


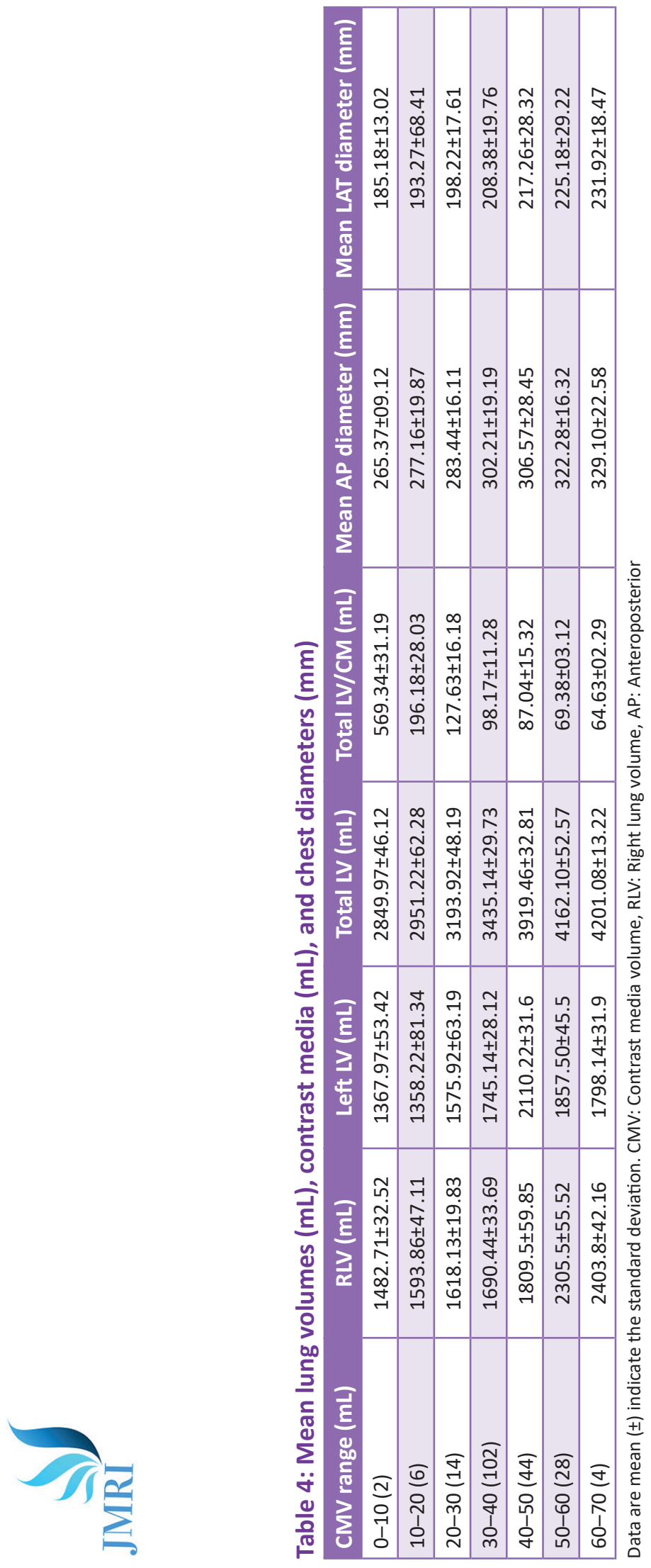

during CT increased double DNA strand breaks which are attributed to increased radiation dose. ${ }^{[32,33]}$ In addition, future studies published should consider the effect of iodinated contrast material on the organ doses administered to patients undergoing $\mathrm{CT}$, as it is important in estimating radiation dose. ${ }^{[34,35]}$ In our study, we demonstrated that increased lung volume was correlated with increased $\mathrm{CMV}$ as well as radiation dose, but when compared to other studies, our CMV range was from 10 to $76 \mathrm{mLs}$ and other studies from 40 to 120 mLs. ${ }^{[36-40]}$ Finally, a strong correlation between the increased lung and contrast volumes demonstrated no correlation with BMI. Therefore, the radiation dose increase can be attributed to the increased volume in the pulmonary circulation at the time of the CTA and not patient weight.

There were shortcomings in the current study. First, whilst every attempt was made to have uniform inspiration between patient to patient, the exact air volume entering the lungs could not be controlled and thus potentially the increase in blood volume could not be determined. Second, we did not measure the effect between weight-based contrast media protocols and the patient-specific contrast

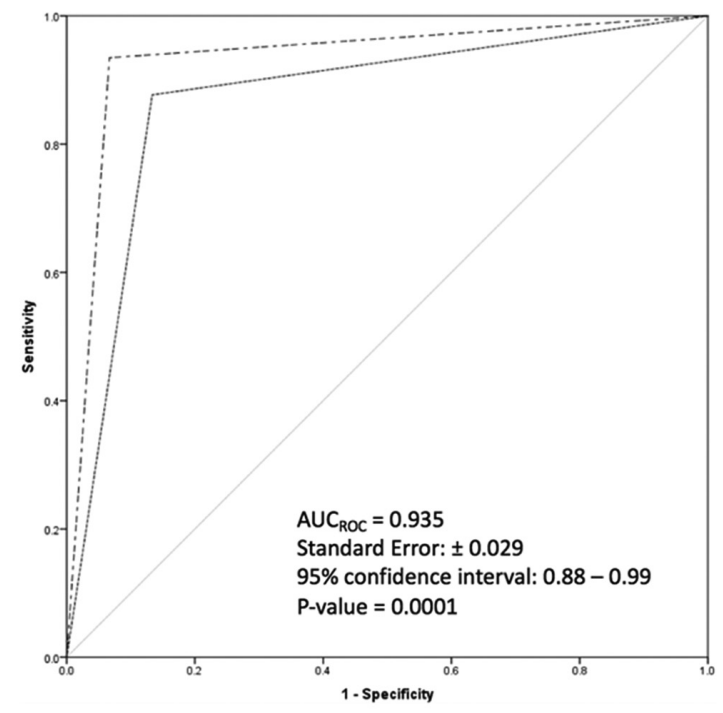

Figure 4: Receiver operating characteristic curve. The graph represents significant sensitivity and specificity in pathology detection at all lung volumes and contrast volumes. Receiver operating characteristic was employed to measure the confidence intervals in pathology detection by radiologists 
Table 5: Contrast media volume ranges

\begin{tabular}{|c|c|c|c|c|c|}
\hline \multirow[t]{2}{*}{ Variables } & \multirow[t]{2}{*}{ All $(n=200)$} & \multicolumn{3}{|c|}{ Contrast media volume range $(\mathrm{mL})$} & \multirow[t]{2}{*}{ P-value } \\
\hline & & $<32(n=60)$ & $33-40(n=81)$ & $>41(n=59)$ & \\
\hline Right lung volume & $1843.42 \pm 9.67$ & $1564.91 \pm 38.31$ & $1749.96 \pm 43.82$ & $2354.65 \pm 98.23$ & 0.007 \\
\hline Left lung volume & $1687.59 \pm 59.17$ & $1434.38 \pm 29.76$ & $1927.68 \pm 14.09$ & $1827.82 \pm 60.64$ & 0.006 \\
\hline Total lung volume & $3530.41 \pm 98.36$ & $2998.65 \pm 47.23$ & $3677.39 \pm 102.44$ & $4181.59 \pm 130.05$ & 0.001 \\
\hline Weight & $78.91 \pm 14.78$ & $78.79 \pm 16.87$ & $77.76 \pm 14.83$ & $80.25 \pm 12.48$ & 0.561 \\
\hline Height & $1.71 \pm 0.09$ & $1.69 \pm 0.09$ & $1.72 \pm 0.08$ & $1.72 \pm 0.09$ & 0.383 \\
\hline Body mass index & $27.07 \pm 4.67$ & $27.53 \pm 5.20$ & $26.31 \pm 4.16$ & $27.37 \pm 4.62$ & 0.383 \\
\hline Anteroposterior length & $298.11 \pm 32.64$ & $275.33 \pm 32.17$ & $304.05 \pm 33.88$ & $286.69 \pm 30.90$ & 0.08 \\
\hline Lateral length & $208.48 \pm 33.15$ & $192.23 \pm 33.15$ & $212.82 \pm 34.99$ & $201.55 \pm 38.24$ & 0.08 \\
\hline Dose length product & $163.90 \pm 49.22$ & $178.83 \pm 61.93$ & $188.38 \pm 40.09$ & $164.44 \pm 38.71$ & 0.08 \\
\hline Effective dose & $2.31 \pm 0.74$ & $1.89 \pm 0.93$ & $2.13 \pm 0.60$ & $2.60 \pm 0.58$ & 0.04 \\
\hline
\end{tabular}

$( \pm)$ Indicate the standard deviation, $P<0.05$, statistically significant with ANOVA

media protocols in pulmonary CTA. Finally, the main limitation of this study is the fact that it is a retrospective one. A randomized clinical trial will be needed to confirm our results.

\section{Conclusion}

High CMVs present a health hazard that could be avoided if patient-specific CMVs are administered. The patient-specific CMVs should preserve image quality, thus maintaining the diagnostic relevance of their usage. A correlation between CMV and lung volume might be important to maintain a good visualization of the pulmonary vasculature. This study showed that administering a patient-tailored CMV can preserve image quality. Moreover, increased CMV is significantly correlated to increased patient lung volume when employing a patient-specific contrast formula. The main limitations of this study are the fact that it is a retrospective study. A randomized clinical trial is needed to confirm our results.

\section{Ethical approval}

All procedures performed in studies involving human partcipants were in accordance with the ethical standards of the American University of Beirut's IRB and with the 1964 Helsinki declaraton and its later amendments or comparable ethical standards. Informed consent was waived since all studies were clinically indicated; patent data contained no identfable informaton and was evaluated retrospectvely.

\section{References}

1. Saade C, Bourne R, El-Merhi F, Somanathan A, Chakraborty D, Brennan P. An optimised patientspecific approach to administration of contrast agent for CT pulmonary angiography. Eur Radiol 2013;23:3205-12.

2. Salameh E, Saade C, Oweis GF. Experimental insight into the hemodynamics and perfusion of radiological contrast in patent and non-patent aortic dissection models. Cardiovasc Eng Technol 2019;10:314-28.

3. Saade C, Hamieh N, Deeb IA, Haddad M, AbiGhanem AS, Ghieh D, et al. An augmented patientspecific approach to administration of contrast agent for CT renal angiography. Int Braz J Urol 2019;45:1022-32.

4. Saade C, Chokr J, Naffaa L, Faraj W, Shamseddine A, Mukherji $\mathrm{D}$, et al. Reduced contrast volume and radiation dose during computed tomography of the pancreas: Timing-specific contrast media protocol. Acad Radiol 2019;26:480-8.

5. Zein-El-Dine S, Bou Akl I, Mohamad M, Chmaisse A, Chahwan S, Asmar K, et al. Split-bolus contrast injection protocol enhances the visualization of the thoracic vasculature and reduced radiation dose during chest CT. Br J Radiol 2018;91:20180509.

6. Saade C, Mohamad M, Kerek R, Hamieh N, Deeb IA, El-Achkar B, et al. Augmented quadruple-phase contrast media administration and triphasic scan protocol increases image quality at reduced radiation dose during computed tomography urography. J Comput Assist Tomogr 2018;42:216-21.

7. Saade C, Mayat A, El-Merhi F. Exponentially decelerated contrast media injection rate combined with a novel patient-specific contrast formula reduces contrast volume administration and radiation dose during computed tomography

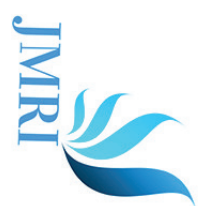


pulmonary angiography. J Comput Assist Tomogr 2016;40:370-4.

8. Saade C, El-Merhi F, El-Achkar B, Kerek R, Vogl TJ, Maroun GG, et al. 256 Slice multi-detector computed tomography thoracic aorta computed tomography angiography: Improved luminal opacification using a patient-specific contrast protocol and caudocranial scan acquisition. J Comput Assist Tomogr 2016;40:964-70.

9. Saade C, Al-Hamra S, Al-Mohiy H, El-Merhi F. Contrast media delivery in the assessment of anomalous left coronary artery from the pulmonary artery. Radiol Technol 2016;87:490-4.

10. Puybasset L, Cluzel P, Gusman P, Grenier P, Preteux F, Rouby JJ. Regional distribution of gas and tissue in acute respiratory distress syndrome. I. Consequences for lung morphology. CT scan ARDS study group. Intensive Care Med 2000;26:857-69.

11. Gimenez S, Teixeira ML, Myashiro R, Carmona MJ, Auler JO Jr., Malbouisson LM. Computed tomography in pulmonary evaluation of children with acyanotic congenital heart defect and pulmonary hyperflow. Rev Bras Anestesiol 2009;59:545-57.

12. Saade C, Bourne R, Wilkinson M, Brennan P. Contrast medium administration and parameters affecting bolus geometry in multidetector computed tomography angiography: An overview. J Med Imaging Radiat Sci 2011;42:113-7.

13. Hittmair K, Fleischmann D. Accuracy of predicting and controlling time-dependent aortic enhancement from a test bolus injection. J Comput Assist Tomogr 2001;25:287-94.

14. You SY, Yoon DY, Choi CS, Chang SK, Yun EJ, Seo YL, et al. Effects of right-versus left-arm injections of contrast material on computed tomography of the head and neck. J Comput Assist Tomogr 2007;31:677-81.

15. Saade C, Bourne R, Wilkinson M, Brennan P. Cardiovascular CTA Applications: Patient-specific Contrast Formulae. United States: Proceedings SPIE; 2013.

16. Huda W, Ogden KM, Khorasani MR. Converting doselength product to effective dose at CT. Radiology 2008;248:995-1003.

17. Brennan PC, McEntee M, Evanoff M, Phillips $P$, O'Connor WT, Manning DJ. Ambient lighting: Effect of illumination on soft-copy viewing of radiographs of the wrist. Am J Roentgenol 2007;188:W177-80.

18. Saade RB, Wilkinson M, Evanoffb M, Brennan P. A reduced contrast volume acquisition regimen based on cardiovascular dynamics improves visualisation of head and neck vasculature with carotid MDCT angiography. Eur J Radiol 2012;82:e64-9.

19. Bath M, Mansson LG. Visual grading characteristics (VGC) analysis: A non-parametric rank-invariant statistical method for image quality evaluation. $\mathrm{Br} \mathrm{J}$ Radiol 2007;80:169-76.
20. McDermott S, Otrakji A, Flores EJ, Kalra MK, Shepard JA, Digumarthy SR. Should dual-energy computed tomography pulmonary angiography replace single-energy computed tomography pulmonary angiography in pregnant and postpartum patients? J Comput Assist Tomogr 2018;42:25-32.

21. Li X, Chen GZ, Zhao YE, Schoepf UJ, Albrecht MH, Bickford MW, et al. Radiation optimized dual-source dual-energy computed tomography pulmonary angiography: Intra-individual and inter-individual comparison. Acad Radiol 2017;24:13-21.

22. Aschoff AJ, Catalano C, Kirchin MA, Krix M, Albrecht T. Low radiation dose in computed tomography: The role of iodine. Br J Radiol 2017;90:20170079.

23. Bae KT. Intravenous contrast medium administration and scan timing at CT: Considerations and approaches. Radiology 2010;256:32-61.

24. Sahebjami H. Dyspnea in obese healthy men. Chest 1998;114:1373-7.

25. Ray CS, Sue DY, Bray G, Hansen JE, Wasserman K. Effects of obesity on respiratory function. Am Rev Respir Dis 1983;128:501-6.

26. Jenkins SC, Moxham J. The effects of mild obesity on lung function. Respir Med 1991;85:309-11.

27. Rubinstein I, Zamel N, DuBarry L, Hoffstein V. Airflow limitation in morbidly obese, nonsmoking men. Ann Intern Med 1990;112:828-32.

28. Bedell GN, Wilson WR, Seebohm PM. Pulmonary function in obese persons. J Clin Invest 1958;37:1049-60.

29. Bass AR, Fields KG, Goto R, Turissini G, Dey S, Russell LA. Clinical decision rules for pulmonary embolism in hospitalized patients: A systematic literature review and meta-analysis. Thromb Haemost 2017;117:2176-85.

30. Milà $M$, Bechini J, Vázquez $A$, Vallejos V, Tenesa $M$, Espinal A, et al. Acute pulmonary embolism detection with ventilation/perfusion SPECT combined with full dose CT: What is the best option? Rev Esp Med Nucl Imagen Mol 2017;36:139-45.

31. Takx RA, Henzler T, Schoepf UJ, Germann T, Schoenberg SO, Shirinova A, et al. Predictive value of perfusion defects on dual energy CTA in the absence of thromboembolic clots. J Cardiovasc Comput Tomogr 2017;11:183-7.

32. Piechowiak El, Peter JF, Kleb B, Klose KJ, Heverhagen JT. Intravenous iodinated contrast agents amplify DNA radiation damage at CT. Radiology 2015;275:692-7.

33. Kuefner M, Brand M, Engert C, Schwab S, Uder M. Radiation induced DNA double-strand breaks in radiology. Röfo 2015;187:872-8.

34. Sahbaee P, Segars WP, Marin D, Nelson RC, Samei E. The effect of contrast material on radiation dose at CT: Part I. Incorporation of contrast material dynamics in anthropomorphic phantoms. Radiology 2017;283:739-48. 
Saade, et al.: Patent specific contrast protocol significantly related to patent lung volume during CTPA

35. Sahbaee P, Abadi E, Segars WP, Marin D, Nelson RC, Samei $E$. The effect of contrast material on radiation dose at CT: Part II-a systematic evaluation across 58 patient models. Radiology 2017;283:749-57.

36. Laqmani A, Kurfurst $M$, Butscheidt $S$, Sehner $S$, Schmidt-Holtz J, Behzadi C, et al. CT pulmonary angiography at reduced radiation exposure and contrast material volume using iterative model reconstruction and iDose4 technique in comparison to FBP. PLoS One 2016;11:e0162429.

37. Kerl JM, Lehnert T, Schell B, Bodelle B, Beeres M, Jacobi $\mathrm{V}$, et al. Intravenous contrast material administration at high-pitch dual-source CT pulmonary angiography: Test bolus versus bolus- tracking technique. Eur J Radiol 2012;81:2887-91.

38. Damm R, Mohnike K, Gazis A, Rogits B, Seidensticker M, Ricke J, et al. Improvement of contrast media enhancement in CTA evaluating pulmonary embolism by utilizing delayed bolus tracking in the descending aorta. Pol J Radiol 2016;81:422.

39. Zapala MA, Zurakowski D, Lee EY. Comparison of mechanical versus hand administration of IV contrast agents for pediatric pulmonary CT angiography. Am J Roentgenol 2017; 208:632-6.

40. Mourits $M$, Nijhof Ws, van Leuken $M$, Jager $G$, Rutten M. Reducing contrast medium volume and tube voltage in CT angiography of the pulmonary artery. Clin Radiol 2016;71:615, e7-13. 